\title{
Drying and shrinkage of computer simulated paper
}

\author{
D. J. Gates* M. Westcott ${ }^{\dagger}$
}

(Received 21 June 2002; revised 10 March 2003)

\begin{abstract}
We describe the modelling and computation of paper shrinkage during drying, using a finite difference method and simulated paper structure. Radial contraction of drying fibres leads to axial compression of crossing, bonded fibres. This microcompression process makes a major contribution to the shrinkage of the paper. The influence of fibre orientation is computed, and shown to be very significant, in accordance with observations. The method relies on the possibility of maintaining fixed anisotropic stiffness constants as the network rotates.
\end{abstract}

${ }^{*}$ CSIRO Mathematical and Information Sciences, PO Box 664, Canberra, ACT 2601, Australia. mailto:david.gates@csiro.au

${ }^{\dagger}$ CSIRO Mathematical and Information Sciences, PO Box 664, Canberra, ACT 2601, Australia. mailto:mark. westcott@csiro.au

${ }^{0}$ See http://anziamj.austms.org.au/V44/E045/home.html for this article, (c) Austral. Mathematical Soc. 2003. Published June 5, 2003 ISSN 14468735 


\section{Contents}

1 Introduction

E270

2 Simulating paper structure

E271

3 Modelling shrinkage

E273

4 Link stiffnesses

E275

5 Numerical solution

E277

6 Influence of fibre orientation

E279

7 Existence and uniqueness of link stiffnesses

E282

References

E284

\section{Introduction}

The casual observer will note that paper expands when wetted and that wet paper shrinks on drying. Shrinkage is also manifested as tension when paper is dried under constraint. It has an important influence on the mechanical properties of paper and of paper board used in fabricating boxes and suchlike. There are also effects of shrinkage and tension in paper making machines.

Free fibres shrink in diameter (radially) but not in length (axially). So why does paper shrink? Page and Tydeman [1], showed that shrinkage occurs at bonded interfaces between fibres. Radial shrinkage of one fibre leads to axial compression of the fibre to which it is bonded, called a microcompression. So the paper network shrinks by an amount determined by the equilibrium between 
tensile and compressive forces at bonded interfaces. It is notable that this bonding is due to the formation of hydrogen bonds at the molecular level, and it quite strong. Page and Tydeman obtained micrographs of bonded fibres that clearly show such microcompressions.

The aim here is to model the microcompression process and thereby quantify the shrinkage of paper in terms of the shrinkage of individual fibres. We shall need to first simulate the paper structure and then develop a finite difference method to compute the shrinkage of this structure.

\section{Simulating paper structure}

We shall ultimately use a 2D model to simulate shrinkage. However, a vital input is the bonding between fibres. This is determined by the interaction between fibres in 3D, so we first simulate a 3D model. Then the influence of fibre dimensions and fibre flexibility can be incorporated in a realistic way $[2,3]$.

A fibre is represented as a flexible, hollow tube [2,3], which is initially straight. Points on the fibre surface are confined to a fine 3-dimensional grid. The grid spacing or cell dimension in the $Z$ direction of the paper (normal to the surface) is equal to the fibre wall thickness. Spacing in the $X, Y$ plane is typically about double the $Z$ spacing. A typical fibre is of order 5 to 10 cells in circumference (that is, of order 10 to 20 times the wall thickness) and of order 100 to 200 cells long, and so occupies of order 1000 cells. The length distribution can be varied.

Fibre centres are chosen randomly and uniformly in a small square, and have angles and lengths chosen randomly from prob- 
ability distributions. The square is a little wider than the longest fibre. Fibres that extend outside the square are mapped periodically back into the square, so the paper is effectively an infinite tiling. This removes edge effects, so that the small square imitates a much larger homogeneous sheet of paper.

An initially straight fibre is allowed to flex so as to conform closely to other fibres. Undulations or 'kinks' in the fibre wall are limited to one $Z$ grid step per $X$ or $Y$ grid step. The first fibre is placed flat on a plane. The next starts straight and level above the plane and is lowered vertically as far as possible, consistent with the kink constraints and the condition that the fibres are nonintersecting sets in 3-dimensions (or non-penetrating solid bodies). Subsequent fibres are deposited in similar fashion.

The hollow interiors of the tubes are called lumens. The structures so generated have collapsed lumens. The simulation then opens lumens where this is consistent with no overlaps and the kink constraints, while confining the structure between two horizontal planes. Then the degree of collapse will vary along the length of the fibre, tending to be greater near fibre crossings. Figure 1 is a section view of a simulation with 200 fibres. Fibre walls are drawn thinner than they actually are so that their individuality is evident. Areas of contact between fibres are then treated as bonded, so one has a complex 3D solid structure.

Some features of Figure 1 warrant comment. Some fibre sections appear isolated, because the fibres that constrain them do not appear in this section. Some fibre sections are not closed, because the fibres are open ended tubes cut at an angle across their ends. Vertical stripes on fibres indicate that a vertical part of the fibre wall lies in the section.

Figure 2 shows 20 inner fibres (numbers 101-120) and their cells 


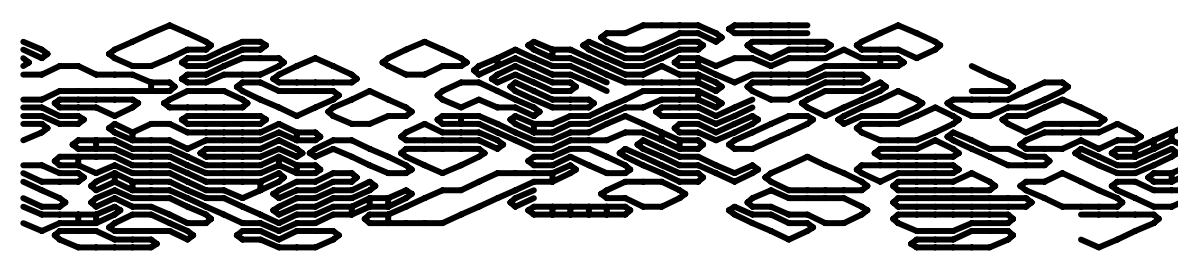

Figure 1: Part of a section view of a simulation with 200 fibres.

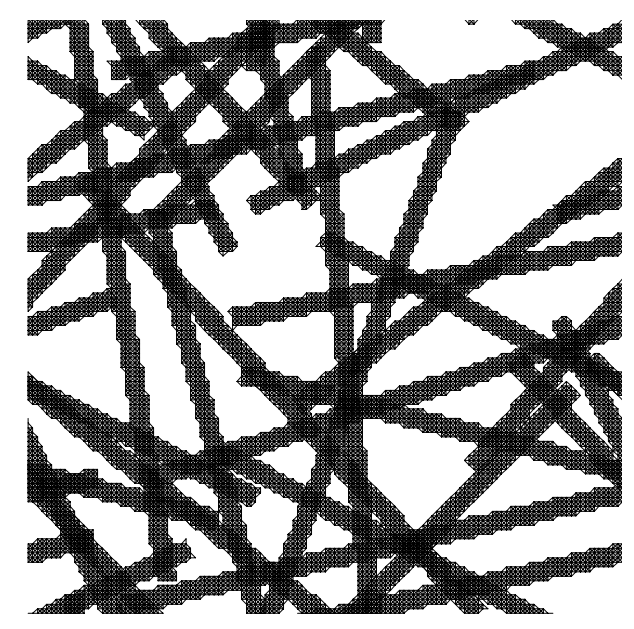

Figure 2: Twenty inner fibres and their cells in the simulation.

in the simulation. Fibre orientation is isotropic in this case.

\section{Modelling shrinkage}

Figures 1 and 2 represent the formed paper when all free water has been removed but water absorbed by fibres remains. The fibres are assumed to have expanded radially by $30 \%$, but not axially, relative to their dry state. In this wet state, fibres have length/width 
ratio 25 .

We regard a fibre as an anisotropic elastic object. Then the simulated paper is a complex elastic structure, resembling a fabricated frame of steel struts. We treat wet fibre expansion as an elastic strain due to internal forces created by water pressure. When water is removed, the fibres relax, but contraction is limited by bonding. The aim is to compute the equilibrium state of the whole bonded network of dry fibres.

The first step is to project the 3D structure on the plane, and treat the deformation as $2 \mathrm{D}$. Thus out-of-plane effects like wrinkling are not included, but these occur on a larger distance scale. The $3 \mathrm{D}$ structure has served the purpose of identifying bond locations. Now one has a 2D structure comprising many superimposed and connected elastic sheets. The equilibrium is computed using a finite difference scheme whose nodes are the projected centres of the existing cells. The periodic boundary conditions are used. Stiffness of fibres is represented by elastic links between these nodes, as in Figure 3. Bonds between fibres become elastic links between bonded nodes, representing the shear stiffness of bonds.

Fibres have stiffness constants $C_{i j k l}, i, j, k, l=1,2$ relating the stress components $\sigma_{i j}$ and strain components $\epsilon_{i j}$

$$
\sigma_{i j}=\sum_{k, l} C_{i j k l} \epsilon_{k l}
$$

with certain symmetries. In another common notation [4], this takes the form

$$
\sigma_{\alpha}=\sum_{\beta} C_{\alpha \beta} \epsilon_{\beta}
$$

with $\alpha, \beta=1,2,6$ (index 3 is reserved for the third dimension) and $\sigma_{1}=\sigma_{11}, \sigma_{2}=\sigma_{22}, \sigma_{6}=\sigma_{12}$ and $C_{\alpha \beta}=C_{\beta \alpha}$. For simplicity, 


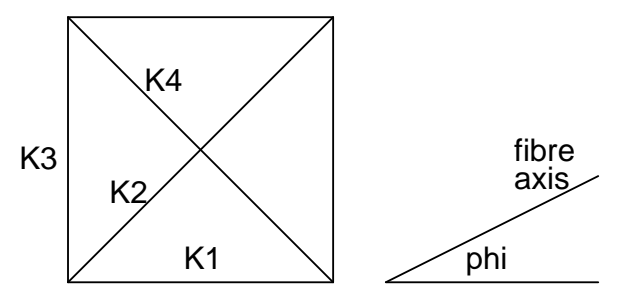

FiguRE 3: Elastic links between nodes.

we use a Cauchy model (1806), in which links are linear springs, freely pivoting at nodes. This formed the basis of Cauchy's atomic theory of elasticity [4]. It implies that the $C_{i j k l}$ are invariant under permutations of $i, j, k, l$, so there are only 5 distinct constants. Equivalently $C_{12}=C_{66}$, which is one of the 'Cauchy relations' [4] (now known not to be generally applicable).

\section{Link stiffnesses}

Every fibre has a different angle $\phi$ relative to the grid, so we have to choose link stiffnesses (not stiffness constants) $K_{b}(\phi), b=1, \ldots, 4$ depending on $\phi$ as in Figure 3. They have dimensions force/length.

We now study the question of whether such $K_{b}(\phi)$ can be chosen so that every fibre has the same stiffness constants relative to its own axes. They are given by the equations of equilibrium

$$
\sum_{b=1}^{4} M_{a b} K_{b}=C_{a}, \quad a=1, \ldots, 5,
$$


with another notation for stiffness constants

$$
\begin{aligned}
& C_{1}=C_{1111}=C_{11}, \\
& C_{2}=C_{1112}=C_{16}, \\
& C_{3}=C_{1122}=C_{12}, \\
& C_{4}=C_{1222}=C_{26}, \\
& C_{5}=C_{2222}=C_{22},
\end{aligned}
$$

and

$$
M_{a b}=r_{b}^{2} \cos ^{5-a} \theta_{b} \sin ^{a-1} \theta_{b},
$$

where $r_{1}=r_{3}=1, r_{2}=r_{4}=\sqrt{2}$ are the lengths of the links and

$$
\theta_{1}=-\phi, \quad \theta_{2}=\pi / 4-\phi, \quad \theta_{3}=\pi / 2-\phi, \quad \theta_{4}=3 \pi / 4-\phi,
$$

are the angles between the fibre axis and the links. In effect, $M_{a b}$ is divided by the unit area of the cell in Figure 3, and is therefore dimensionless. The $C_{i}$, being $2 \mathrm{D}$ stiffness constants have dimensions force/length.

We have $5 \times(0, \pi / 2)$ equations in $4 \times(0, \pi / 2)$ unknowns $K_{b}(\phi)$. This comprises an uncountable infinity of equations in an uncountable infinity of unknowns, where the number of equations exceeds the number of unknowns by an uncountable infinity. The question of interest is whether these equations have any solutions. As shown in Section 7, a solution exists for all $\phi$ if and only if

$$
C_{2}=C_{4}, \quad C_{1}+C_{5}=6 C_{3},
$$

and the solution is then unique. By simple elimination, the solution is found to be

$$
\begin{aligned}
& K_{1}=(A+B \cos 2 \phi-D \sin 2 \phi) / 2, \\
& K_{2}=(A+B \sin 2 \phi+D \cos 2 \phi) / 4, \\
& K_{3}=(A-B \cos 2 \phi+D \sin 2 \phi) / 2, \\
& K_{4}=(A-B \sin 2 \phi-D \cos 2 \phi) / 4,
\end{aligned}
$$


where

$$
A=\left(C_{1}+2 C_{3}+C_{5}\right) / 2, \quad B=C_{1}-C_{5}, \quad D=2\left(C_{2}+C_{4}\right) .
$$

Furthermore $K_{b} \geq 0$ if and only if $A^{2} \geq B^{2}+D^{2}$, which reduces to

$$
4 C_{2}^{2} \leq\left(C_{1}-C_{3}\right)\left(C_{5}-C_{3}\right) .
$$

Thus (7) and (10) are the conditions under which one can have the same stiffness constants for every fibre (every orientation of the grid).

There is evidence that fibres are approximately 'orthotropic'; a common symmetry that implies $C_{2}=C_{4}=0$, leaving only 2 independent constants. Then the constraints reduce to

$$
C_{12}=\left(C_{11}+C_{22}\right) / 6, \quad C_{11} / 5 \leq C_{22} \leq 5 C_{11},
$$

which put limits on the degree of elastic anisotropy of a fibre. We take relative values suggested by data: $C_{11}=1.5, C_{22}=0.4$ and $C_{12}=0.31 \dot{6}$. Units are not required because we shall compute only deformations, not forces.

Shear stiffness of bonds is comparable to fibre wall stiffness, so we take a value 1 . Much larger values give similar overall shrinkage. (See a small shear displacement of bonds in Figure 5).

\section{$5 \quad$ Numerical solution}

The elastic equilibrium is computed via Gauss-Seidel iteration. Figure 4 shows a small area of wet paper from Figure 2. Figure 5 shows the state of fibres from Figure 4 after drying. Where fibres are not bonded they have shrunk radially by up to $30 \%$, so that squares in 


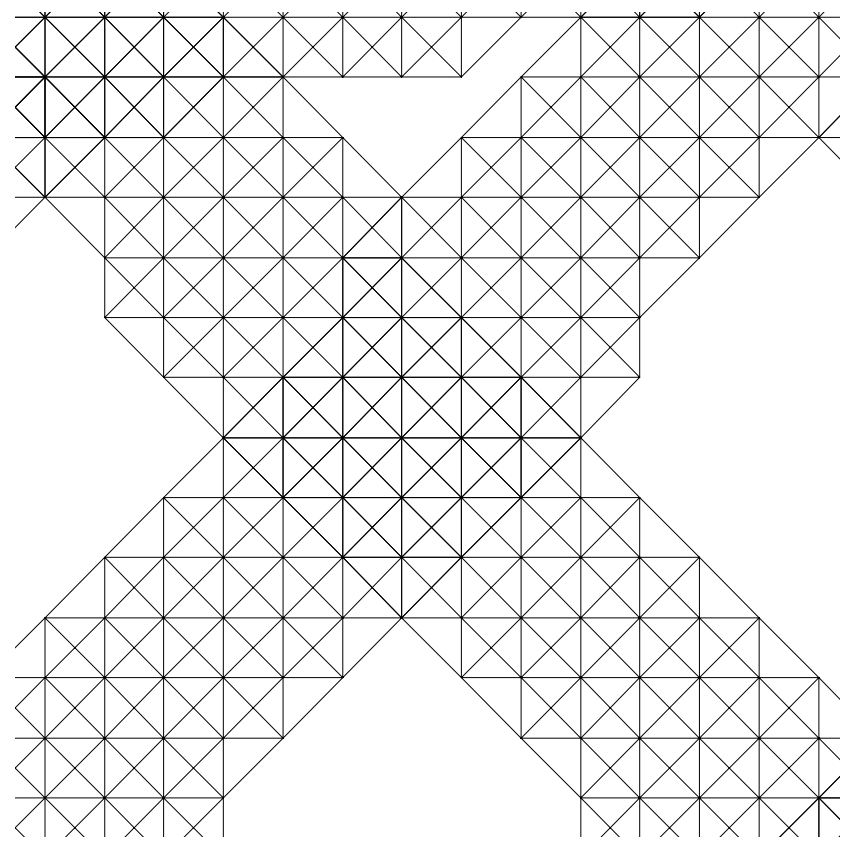

Figure 4: A small area of wet paper from Figure 2. 
the mesh have become diamonds. But, overall, the radial shrinkage of fibres is substantially less than $30 \%$ and is variable along their lengths, due to bonding with fibres not shown. The shrinkage of the whole structure is only $6 \%$ in both directions in the plane. Comparing the dimensions of the elements in the bonded region of the 2 crossing fibres in Figure 5, one sees that both fibres have experienced both radial and axial contractions of around $10 \%$. The axial contractions represent the microcompressions described in Figure 1. There is evidently much residual stress locked into the dry structure. Fibres are generally in a state of radial tensile stress and axial compressive stress. Features of the deformation of fibres in the dry paper are discussed in heuristic terms in [1], but even in our simplified model, the deformations are too complex to summarize adequately in a verbal description.

Computation time depends on the initial state for the iteration. If one takes an initial solution with no shrinkage, then the iteration could take up to an hour to converge adequately on a SUN ULTRA 10. If one has an independent estimate of shrinkage, then the initial state for the iteration is taken as uniformly contracted with this shrinkage. If this estimate is good, then the computation involves mainly local strains, and computation time is much smaller.

\section{$6 \quad$ Influence of fibre orientation}

The simulated drying process was carried out also when fibres have orientation parameter $K=0.5$ and 1.5 in the von Mises angular distribution

$$
g(\phi)=\frac{1}{\pi I_{0}(K)} \exp (K \cos 2 \phi),
$$




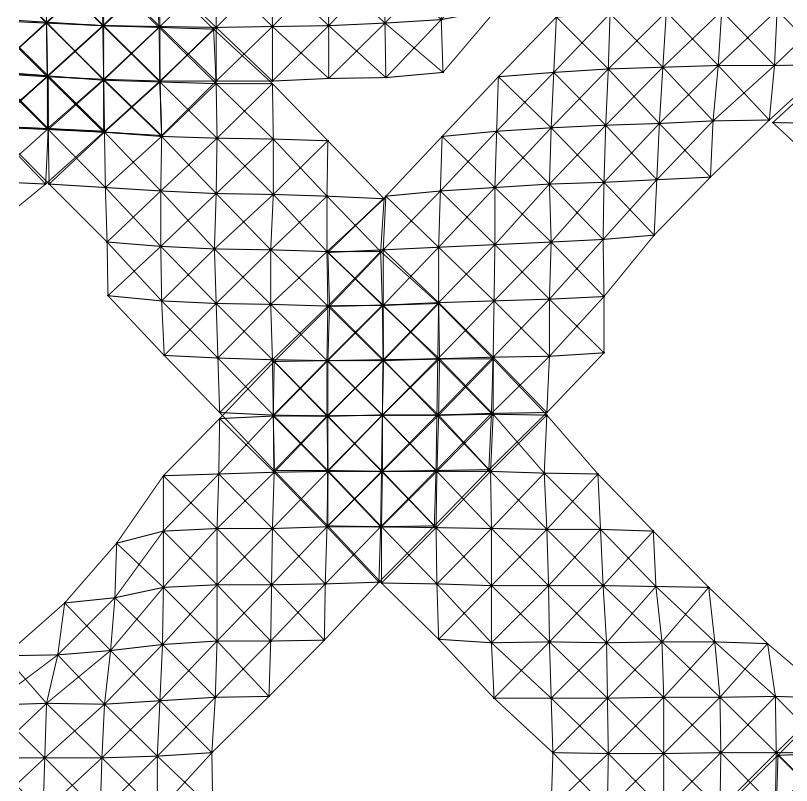

FiguRE 5: The state of fibres from Figure 4 after drying. 
TABLE 1: Dependence of directional shrinkage on fibre orientation.

\begin{tabular}{cccc}
\hline \multicolumn{2}{c}{ orientation } & \multicolumn{2}{c}{ \% shrinkage } \\
$K$ & $Q$ & MD & CD \\
\hline 0 & -0.004 & 6 & 6 \\
0.5 & 0.243 & 5 & 8 \\
1.5 & 0.596 & 3 & 12 \\
\hline
\end{tabular}

for fibres making angle $-\pi / 2 \leq \phi \leq \pi / 2$ with the machine direction (MD). For $K=0$ the fibre angles are isotropic, whereas $K=0.5$ is a typical value for machine-made paper, and $K=1.5$ is the highest orientation observed by Perkins and Mark [5]. A more intuitive measure of fibre orientation is the quantity

$$
Q=\frac{1}{N_{f}} \sum_{i} \cos \left(2 \phi_{i}\right)
$$

where $\phi_{1}, \ldots, \phi_{N_{f}}$ are the fibre angles relative to the MD. Thus $Q$ has expectation value $I_{1}(K) / I_{0}(K)$, which is 0 for isotropic orientation and 1 for fibres all aligned in the MD. The simulation results are are given in Table 1.

As expected, MD shrinkage is reduced and cross direction (CD) shrinkage increased as a result of fibre orientation. This is in general agreement with measurements [1].

The important topic of drying tension and drying restraint could be investigated using the present methods. The implications for stiffness and strength of the dry paper could then be investigated. 


\section{Existence and uniqueness of link stiffnesses}

To prove eq. (7) it is convenient to consider a natural generalization of eq. (3):

$$
\mathbf{A x}=\mathbf{b}
$$

with $\mathbf{A}=\left[a_{i j}\right]$ where

$$
a_{i j}=\left(\cos \theta_{j}\right)^{n+1-i}\left(\sin \theta_{j}\right)^{i-1}
$$

with $i=1, \ldots, n+1, j=1, \ldots, n$ and $\theta_{j}=(j-1) \pi / n-\phi$. Here we investigate conditions for existence and uniqueness of solutions of (14).

Let $\mathbf{S}_{n}$ be the $n \times n$ matrix formed by the first $n$ rows of $\mathbf{A}$. Provided no $\cos \theta_{j}$ is zero,

$$
\operatorname{det} \mathbf{S}_{n}=\operatorname{det} \mathbf{T}_{n} \prod_{j=1}^{n}\left(\cos \theta_{j}\right)^{n}
$$

where $\mathbf{T}_{n}=\left[t_{i j}\right]$ with $t_{i j}=\left(\tan \theta_{j}\right)^{i-1}$, whose determinant is of Vandermonde type with value

$$
\operatorname{det} \mathbf{T}_{n}=\prod_{1 \leq i<j \leq n}\left(\tan \theta_{j}-\tan \theta_{i}\right)
$$

It follows that

$$
\operatorname{det} \mathbf{S}_{n}=\left[\prod_{j=1}^{n} \cos \theta_{j}\right]\left[\prod_{1 \leq i<j \leq n} \sin \left(\theta_{j}-\theta_{i}\right)\right],
$$

regardless of whether any $\cos \theta_{j}$ is zero. No $\sin \left(\theta_{j}-\theta_{i}\right)$ is ever zero because $\theta_{j}-\theta_{i}=(j-i) \pi / n$. If $\phi$ is such that no $\cos \theta_{j}$ is zero, then $\mathbf{S}_{n}$ is non-singular and so $\mathbf{A}$ has rank $n$. 
Evidently only one $\cos \theta_{j}$ can be zero for a fixed $\phi$. Suppose this is the case $j=n$. Then let $\mathbf{P}$ be the $n \times n$ matrix obtained by deleting the $n$th row of $\mathbf{A}$. Now

$$
\operatorname{det} \mathbf{P}=\sin ^{n} \theta_{n} . \operatorname{det} \mathbf{S}_{n-1} .
$$

But $\sin \theta_{n} \neq 0$ for this $\phi$ and, by the above argument, $\operatorname{det} \mathbf{S}_{n-1}$ is non-zero for this $\phi$. Thus $\mathbf{P}$ is non-singular and so again $\mathbf{A}$ has rank $n$. Likewise if any other $\cos \theta_{j}=0$, so solutions are always unique.

For fixed $\phi$, solutions exist if $\operatorname{rank}[\mathbf{A} \mid \mathbf{b}]=n$, where $[\mathbf{A} \mid \mathbf{b}]$ is the $(n+1) \times(n+1)$ augmented matrix. Write the rows of $\mathbf{A}$ as $\mathbf{r}_{i}$, $i=1, \ldots, n+1$. Since $\operatorname{rank} \mathbf{A}=n$, there must be a unique (up to a scale factor) linear relation between the $\mathbf{r}_{i}$, say

$$
\sum_{i=1}^{n+1} \lambda_{i} \mathbf{r}_{i}=\mathbf{0} .
$$

where the $\lambda_{i}$ are not all zero. If $\operatorname{rank}[\mathbf{A} \mid \mathbf{b}]=n$ too, there must be the same linear relation between its rows, because of the uniqueness of (20). Then (20) implies

$$
\sum_{i=1}^{n+1} \lambda_{i} b_{i}=0 .
$$

This is the single constraint on $\mathbf{b}$ that gives a unique solution of (14), for fixed $\phi$. But the $\lambda_{i}$ depend on $\phi$, so (21) represents an uncountable infinity of constraints, parametrised by $\phi$.

For the case $n=4$ one finds that (20) takes the form

$$
\left(\mathbf{r}_{1}-6 \mathbf{r}_{3}+\mathbf{r}_{5}\right) \sin 4 \phi=4\left(\mathbf{r}_{4}-\mathbf{r}_{2}\right) \cos 4 \phi,
$$

whence (21) becomes

$$
\left(b_{1}-6 b_{3}+b_{5}\right) \sin 4 \phi=4\left(b_{4}-b_{2}\right) \cos 4 \phi,
$$


for all $\phi$. If $\phi$ is an even multiple of $\pi / 8$, this implies

$$
b_{4}-b_{2}=0,
$$

and if $\phi$ is an odd multiple of $\pi / 8$, it implies

$$
b_{1}-6 b_{3}+b_{5}=0 .
$$

For all other $\phi,(23)$ implies both (24) and (25), which are just eqs. (7). From another point of view, the equations are satisfied for all $\phi$ if they are satisfied for just 2 values of $\phi$. Hence we have effectively 10 equations in 8 unknowns, requiring 2 constraints.

Acknowledgements: We thank R. S. Anderssen and F. de Hoog for helpful advice, and CRC colleagues for helpful information and discussions. Originally, the work was funded through the CRC for Hardwood Fibre and Paper Science.

\section{References}

[1] D. H. Page and P. A. Tydeman. A new theory of shrinkage, structure and properties of paper. In F. Bolam, editor, The formation and structure of paper, pages 397-425. William Clowes, London, 1962 E270, E279, E281

[2] N. Nilsen, M. Zabihian and K. Niskanen. KCL-PAKKA: a tool for simulating paper properties. Tappi J., 81(5):163-166, 1998. E271

[3] D. J. Gates and M. Westcott. Predicting fibre contact in a 3D model of paper. J. Statistical Physics 94(1/2):31-51, 1999. E271 
[4] A. E. H. Love. A treatise on the mathematical theory of elasticity. New York, Dover, 1944. E274, E275

[5] R. W. Perkins and R. E. Mark. Some new concepts of the relation between fibre orientation, fibre geometry, and mechanical properties. In The Role of Fundamental Research in Papermaking, Trans. Cambridge Symposium, September 1981. Mech. Eng. Pub. Ltd. London, 1983. E281 